\title{
Underlap Counterdoping as an Efficient Means to Suppress Lateral Leakage in the Electron-Hole Bilayer Tunnel FET
}

\author{
C Alper ${ }^{1}$, P Palestri ${ }^{2}$, J L Padilla ${ }^{1}$, A M Ionescu ${ }^{1}$ \\ ${ }^{1}$ Nanoelectronic Devices Laboratory, Ecole Polytechnique Fédérale de Lausanne, \\ Lausanne CH-1015, Switzerland \\ 2 DIEG, University of Udine, Via delle Scienze 206, 33100 Udine, Italy \\ E-mail: cem.alper@epfl.ch
}

\begin{abstract}
The Electron-Hole Bilayer Tunnel FET has been proposed as a Density of States (DOS) switch capable of achieving a subthreshold slope lower than $60 \mathrm{mV} /$ decade at room temperature; however, one of the main challenges is the control of the lateral band-to-band tunneling (BTBT) leakage in the OFF state. In this work, we show that by using oppositely doped underlap regions; the unwanted penetration of the wavefunction into the underlap region at low gate biases is prevented; thereby drastically reducing the lateral BTBT leakage without any penalty on the ON current. The method is verified using a full-quantum 2-D Schrödinger-Poisson solver under the effective mass approximation. For a channel thickness of $10 \mathrm{~nm}$, an $\mathrm{In}_{0.53} \mathrm{Ga}_{0.47} \mathrm{As}$ EHBTFET with counterdoping can exhibit an ON-current up to $20 \mu \mathrm{A} / \mu \mathrm{m}$ and an average subthreshold swing (SS) of about $30 \mathrm{mV} / \mathrm{dec}$. Compared to previous lateral leakage suppression solutions, the proposed method can be fabricated using templateassisted selective epitaxy.
\end{abstract}

\section{Introduction}

The relentless drive that governed the scaling of MOSFETs has started to get close to the fundamental limits imposed by the device working principle. One such limitation applies to the so-called subthreshold swing (SS) which is limited to $60 \mathrm{mV} / \mathrm{dec}$ at room temperature for MOSFETs due to thermionic emission of carriers [1]. In order to overcome this incompressible barrier, Tunnel FETs (TFET) have been proposed and studied as a promising candidate [2]. TFETs have a demonstrated potential [3] to have $\mathrm{SS}<60 \mathrm{mV}$ and therefore could be a feasible device in low supply voltage applications. Recently, a specific type of TFET called electron-hole bilayer TFET (EHBTFET, see Fig. 1(a,b)) was proposed and was shown to have very promising characteristics [4] using semiclassical simulations. The same device was then simulated using a quantum mechanical method which describes the conduction mechanism as subband-to-subband tunneling between electron and hole 2D gases [5]. Later studies $[6,7]$ showed that a leakage path exists in the EHBTFET due to the penetration of the wavefunction (WF)

Published in Semic. Sci tech, v.31, n.4, p.045001 
into the so-called underlap region (see Fig. 1(c)). This is due to the field-induced quantization being considerably weaker in the underlap region which is controlled by only one gate. This WF penetration causes significant lateral leakage [6] with subsequent SS degradation.

Recently, the use of a hetero-gate configuration to align the subband energy in the underlap region to the one in the overlap region was proposed and was shown to have excellent characteristics [7]. In this work, we propose an alternative method based on the introduction of counterdoped pockets in the underlap regions (Fig. 1(d)) to achieve a similar effect. Compared to the hetero-gate method, the use of counterdoping does not require utilizing four different metal workfunctions in the device and opens the possibility to fabricate the EHBTFET using specific growth methods [8].

\section{Simulation Methodology}

We utilize a 2-D full-quantum Schrödinger-Poisson solver based on the parabolic effective mass approximation (EMA) [6] for simulations including lateral leakage. Finite Element Method (FEM) is used to discretize the partial differential equation system. Non-linear scheme is used to couple Schrödinger and Poisson equations [9]. When computing the charge, the source (drain) Fermi level is used for holes (electrons), assuming that the BTBT does not have a significant effect on the carrier distribution with respect to the equilibrium condition. Once the self-consistency is achieved, band-to-band tunneling (BTBT) is calculated as a post-processing step using a model based on the Kane's twoband dispersion [10,11]. This BTBT modeling approach offers an advantage compared to semi-classical models because it directly makes use of the solutions of the Schödinger equation, thereby inherently including the quantization effects on both the WFs and the density of states. The overall BTBT current is given by discrete summation of the contributions from all the possible electron and hole subband pairs (with energies $E_{k \Gamma}$, $E_{k^{\prime} \alpha^{\prime}}$ and WFs $\psi_{k^{\prime} \alpha^{\prime}}, \psi_{k \Gamma}$, respectively). The coupling element between the electron and holes state is given as $[10,12]$ :

$$
M_{c v}=e \sqrt{\frac{\hbar^{2}}{4 \bar{m} E_{G}^{\Gamma}}} \delta_{k_{\perp}, k_{\perp}^{\prime}} \int \psi_{k^{\prime} \alpha^{\prime}}^{*}(\vec{r})|F(\vec{r})| \psi_{k \Gamma}(\vec{r}) d^{2} \vec{r}
$$

Here $|F(\vec{r})|=\sqrt{F_{z}(\vec{r})^{2} C_{0 z}(\theta, \phi)+F_{x}(\vec{r})^{2} C_{0 x}(\theta, \phi)}$ is the modified magnitude of the electric field, $C_{0 x, z}$ are the form factors that account for the polarization dependence of the coupling element $[12,13]$. The $1-\mathrm{D}$ version of the model has been verified against full quantum simulator in [11] and an overall consistency is seen between the 1-D and 2-D models for uniform structures [12]. Examining Eq. 1 it is easily seen that the spatial overlap of the WFs has utmost importance in determining the BTBT current [14], therefore the potential profile of the device needs to be carefully designed to prevent excessive WF overlap in the OFF state.

Since we are solving the Schrödinger equation using closed boundaries, discarding the stationary states that do not contribute to BTBT current is necessary in the post 
processing step. Stationary states are determined by calculating, for each state, the portion of the WF residing in the source or drain regions; and only those states that have sufficient percentage ( $10 \%$ has proved to be a reasonable choice) are included in the BTBT calculation. Stationary states still need to be included in electrostatics calculations to obtain the correct potential profile [15].

\section{Device Structure}

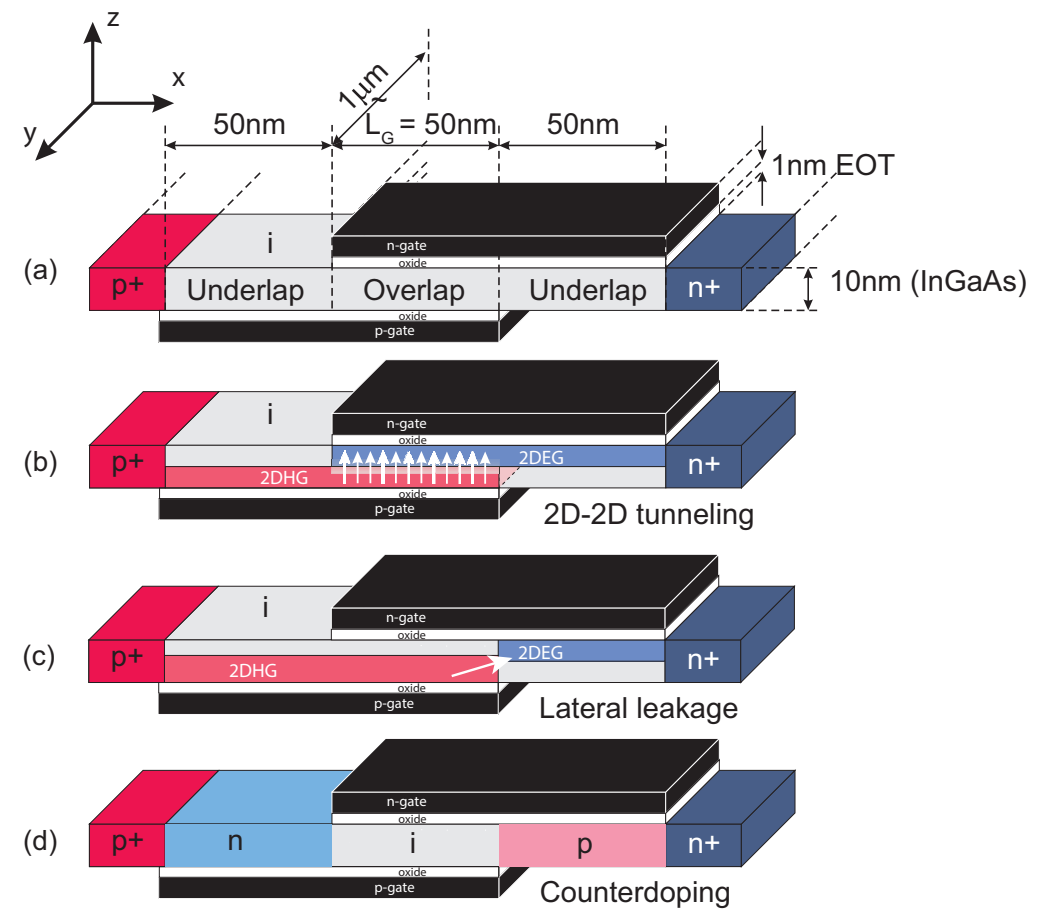

Figure 1. (a) EHBTFET device structure and regions. (b) ON state which occurs when the quantized energies for the 2DEG and 2DHG align; the arrows indicate the BTBT direction. (c) Lateral leakage mechanism at the OFF state. (d) The proposed solution utilizing doped underlap regions (counterdoping).

We simulate an $\operatorname{In}_{0.53} \mathrm{Ga}_{0.47}$ As EHBTFET oriented along the [100] direction. Since InGaAs is a direct gap material, only direct BTBT is included. The bandgap is $0.81 \mathrm{eV}$ and the effective masses are $0.042 \mathrm{~m}_{0}, 0.0503 \mathrm{~m}_{0}$ and $0.465 \mathrm{~m}_{0}$ for electrons, light holes and heavy holes, respectively [16, 17]. The gate oxide has EOT=1nm at both interfaces, which corresponds to roughly 5.9nm of $\mathrm{HfO}_{2}(\epsilon=23)$. We have checked that the magnitude of the electric field inside the oxide is well below the breakdown field for $\mathrm{HfO}_{2}\left(E_{b d} \sim 4 \mathrm{MV} / \mathrm{cm}\right)$ reported in [18]. The source and drain regions are doped p-type and n-type respectively with a doping level of $10^{20} \mathrm{~cm}^{-3}$. We have checked the impact of incomplete activation of dopants in the source and drain regions and seen that the device characteristics are not affected. We assume midgap workfunctions for both n-gate and p-gates. Unless otherwise stated, a $\mathrm{V}_{\mathrm{DS}}=0.25 \mathrm{~V}$ is used. 


\section{Results \& Discussion}

The simulation and device optimization strategy is as follows: first the 1-D version of the quantum mechanical simulator [5] is utilized to perform a parameter sweep on the channel thickness while fixing the both oxide thicknesses at 1nm EOT, as given in Fig. 2. For this figure, n-gate and p-gate biases are swept simultaneously in opposite polarities (i.e. $\mathrm{V}_{\mathrm{p} \text {-gate }}=-\mathrm{V}_{\mathrm{n} \text {-gate }}$ ). The figures of merit that are of interest are the subband alignment voltage $\left(\mathrm{V}_{\text {align }}=\mathrm{V}_{\mathrm{n} \text {-gate }}-\mathrm{V}_{\mathrm{p} \text {-gate }}\right)$ and the $\mathrm{ON}$ current (defined as $I_{D}$ at $\left.\mathrm{V}_{\mathrm{n} \text {-gate }}=\frac{\mathrm{V}_{\text {align }}}{2}+0.25 \mathrm{~V}\right)$. The figure shows the alignment voltage and ON current levels obtained for different channel thicknesses $\mathrm{T}_{\mathrm{CH}}$. It is seen that $\mathrm{T}_{\mathrm{CH}}=10 \mathrm{~nm}$ seems to offer a reasonable compromise between the alignment voltage (with a required bias of about $3.3 \mathrm{~V}$ ) and the $\mathrm{ON}$ current (about $10 \mu \mathrm{A} / \mu \mathrm{m}$ ). For the following results, unless otherwise stated, a channel thickness of $10 \mathrm{~nm}$ is used.

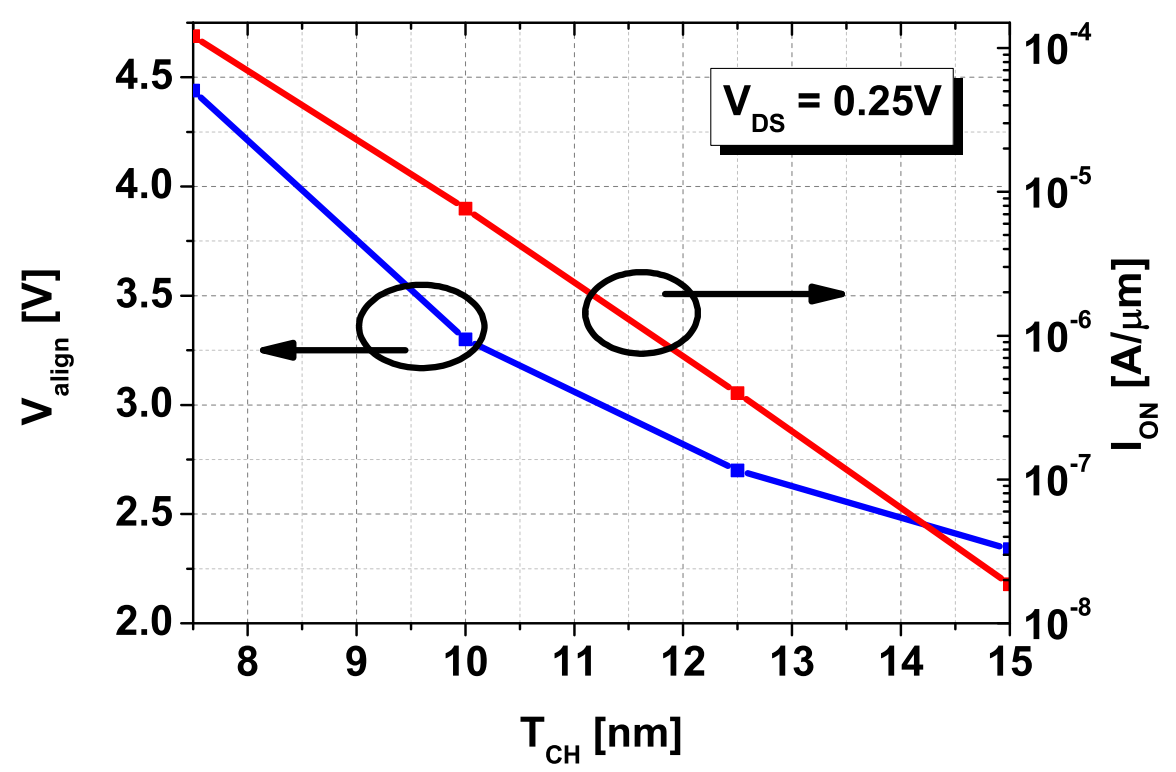

Figure 2. Alignment voltage $\mathrm{V}_{\text {align }}$ and $\mathrm{ON}$ current levels for $\operatorname{In}_{0.53} \mathrm{Ga}_{0.47} \mathrm{As}$ EHBTFET with different channel thicknesses $\mathrm{T}_{\mathrm{CH}}$.

The main idea of using counterdoping in the underlap region is to partially screen the influence of the gate. Fig. 3 reports conduction band profile cut along the $x$ direction at the middle of the channel. Using counterdoping results in a significant increase of the energy barrier for both holes and electrons in their respective underlap regions, which delays the onset of the parasitic lateral tunneling component. Note that counterdoping alleviates the short channel effects (SCE) in the overlap region, by flattening the potential profile around the overlap-underlap boundary. Due to this, counterdoping is also expected to help with the lateral scaling of the EHBTFET by suppressing the SCE. For all the figures shown in this paper, the counterdoping is applied only for the underlap region at the drain side (p-doped region in Fig. 1(d)); whereas the underlap region at the source side is left undoped. This is due to the fact 
that for the devices shown here, we use a p-gate bias that already induces the 2DHG in the overlap region, therefore only the penetration of the electrons needs to be suppressed.

The effect of the increase in energy barrier is easily observed in the BTBT generation rates given in Fig. 4, which indicate orders of magnitude lower rates for counterdoped EHBTFET in the OFF state $\left(\mathrm{G}_{\mathrm{BTBT} \text {,max }} \sim 10^{46} \mathrm{~m}^{-3} \mathrm{~s}^{-1}\right.$ with no counterdoping, compared to $\mathrm{G}_{\mathrm{BTBT} \text {,max }} \sim 10^{34} \mathrm{~m}^{-3} \mathrm{~s}^{-1}$ with counterdoping) which shows the efficiency of the method.

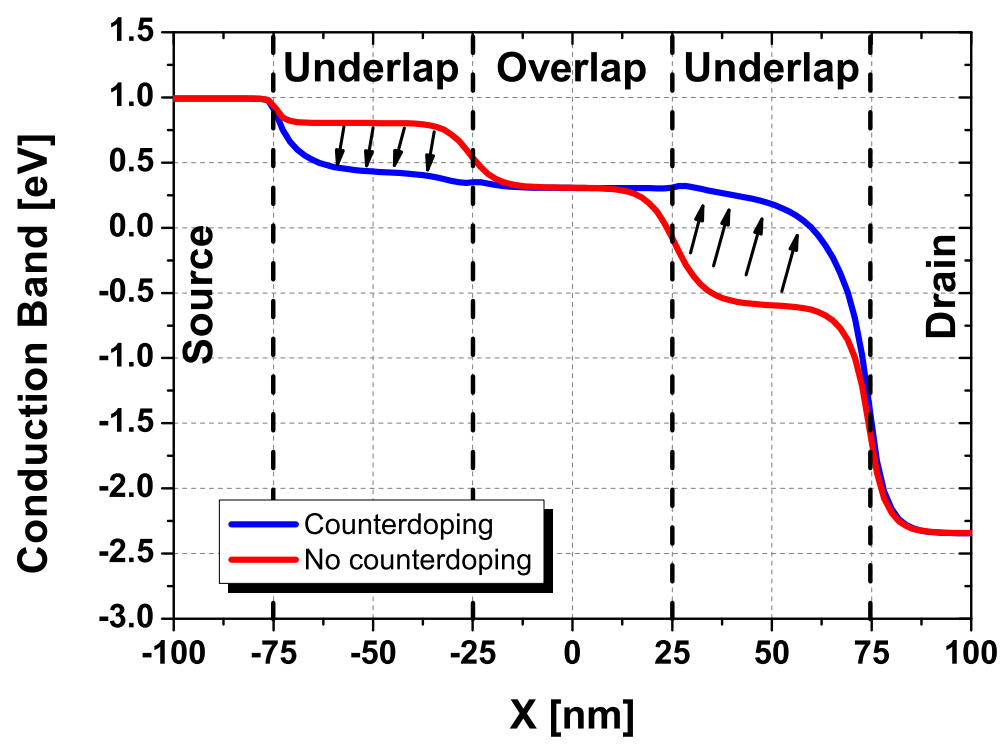

Figure 3. Conduction band profile cut through the middle of the channel along the $\mathrm{x}$ direction for EHBTFETs with (blue) and without (red) counterdoping $\left(\mathrm{N}_{\mathrm{UL}}=1.5 \times 10^{19} \mathrm{~cm}^{-3}\right.$ at both underlap regions $)$ in the OFF state $\left(\mathrm{V}_{\mathrm{n}-\text { gate }}=1.4 \mathrm{~V}, \mathrm{~V}_{\mathrm{p}-\text { gate }}=-1.4 \mathrm{~V}, \mathrm{~V}_{\mathrm{DS}}=0.25 \mathrm{~V}\right)$.

Fig. 5 shows the transfer characteristics using different underlap doping levels (denoted as $\mathrm{N}_{\mathrm{UL}}$ ), where the p-gate bias is fixed at $-1.4 \mathrm{~V}$ while sweeping the n-gate. In the optimized case, $\mathrm{N}_{\mathrm{UL}}=1.5 \times 10^{19} \mathrm{~cm}^{-3}$, an $\mathrm{ON}$ current of about $20 \mu \mathrm{A} / \mu \mathrm{m}$ and an average $\mathrm{SS}$ of $31 \mathrm{mV} /$ dec over eight decades of current are obtained in this particular device. The OFF current is taken to be $0.1 \mathrm{pA} / \mu \mathrm{m}$ (at the n-gate voltage $\mathrm{V}_{\mathrm{OFF}}$ ) and the $\mathrm{ON}$ current is the drain current at $\mathrm{V}_{\mathrm{n}-\text { gate }}=\mathrm{V}_{\mathrm{OFF}}+0.25 \mathrm{~V}$.

The optimum doping is expected to depend on the channel thickness, as the latter impacts the degree of quantization. To investigate this point, another InGaAs EHBTFET with $\mathrm{T}_{\mathrm{CH}}=7.5 \mathrm{~nm}$ is simulated and the transfer characteristics are reported in Fig. 6 using different underlap doping levels. For these set of curves, the pgate bias is fixed at $-2.0 \mathrm{~V}$ while sweeping the n-gate. It is seen that the optimum doping level which suppresses the lateral leakage while preserving the ON current is $\mathrm{N}_{\mathrm{UL}}=3.5 \times 10^{19} \mathrm{~cm}^{-3}$, which is drastically higher than the value found for $\mathrm{T}_{\mathrm{CH}}=10 \mathrm{~nm}$ although the value may slightly differ in different biasing configurations. The optimized configuration in this case yields slightly improved (compared to 10nm) SS and ON 
(a)

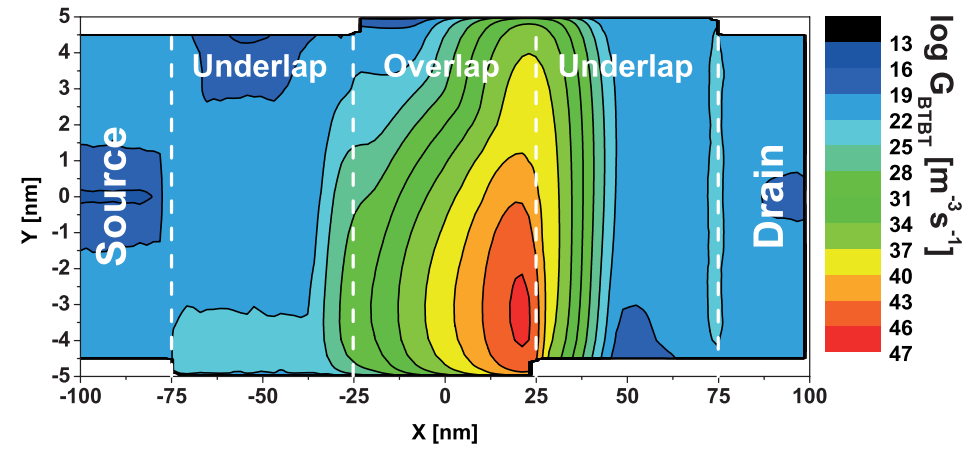

(b)

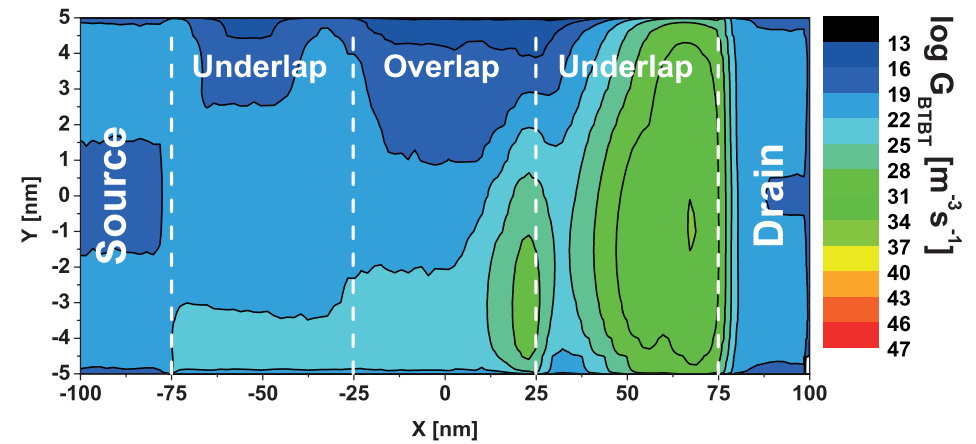

Figure 4. BTBT generation rates for the OFF state $\left(\mathrm{T}_{\mathrm{CH}}=10 \mathrm{~nm}, \mathrm{~V}_{\mathrm{n}-\text { gate }}=1.4 \mathrm{~V}, \mathrm{~V}_{\mathrm{p}-\text { gate }}=-1.4 \mathrm{~V}, \mathrm{~V}_{\mathrm{DS}}=0.25 \mathrm{~V}\right)$ of the InGaAs EHBTFET (a) without counterdoping (b) with counterdoping $\left(\mathrm{N}_{\mathrm{UL}}=1.5 \times 10^{19} \mathrm{~cm}^{-3}\right)$.

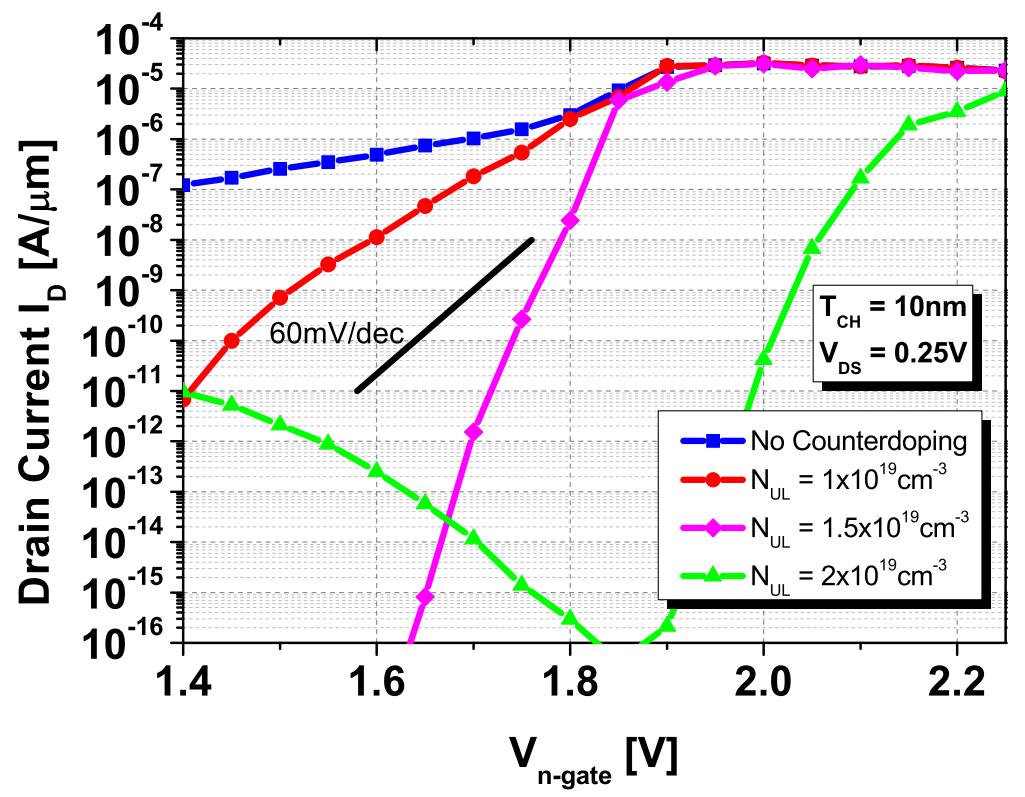

Figure 5. Transfer characteristics for the EHBTFET with different underlap doping levels with $\mathrm{T}_{\mathrm{CH}}=10 \mathrm{~nm}$. $\mathrm{V}_{\mathrm{DS}}=0.25 \mathrm{~V}, \mathrm{~V}_{\mathrm{p} \text {-gate }}=-1.4 \mathrm{~V}$. Note the sudden increase in OFF current for the $\mathrm{N}_{\mathrm{UL}}=2 \times 10^{19} \mathrm{~cm}^{-3}$ curve, caused by a second leakage path.

current values $\left(\mathrm{I}_{\mathrm{ON}}=100 \mu \mathrm{A} / \mu \mathrm{m}\right.$ and an average SS of $27 \mathrm{mV} /$ dec over ten decades of current). Similarly, thicker channels require less doping e.g. the optimum doping is 


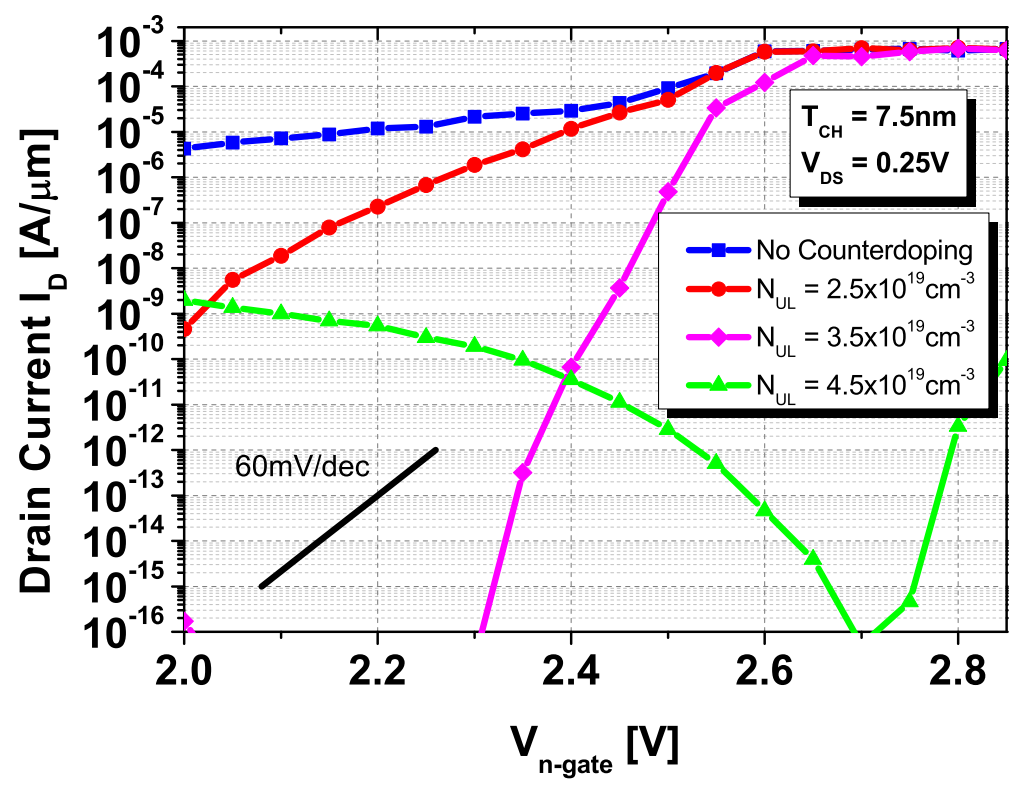

Figure 6. Transfer characteristics for the EHBTFET with different underlap doping levels with $\mathrm{T}_{\mathrm{CH}}=7.5 \mathrm{~nm}$. $\mathrm{V}_{\mathrm{DS}}=0.25 \mathrm{~V}, \mathrm{~V}_{\mathrm{p}-\text { gate }}=-2 \mathrm{~V}$. An increase in OFF current similar to Fig. 5) is obverved for $\mathrm{N}_{\mathrm{UL}}=4.5 \times 10^{19} \mathrm{~cm}^{-3}$.

$\mathrm{N}_{\mathrm{UL}}=5 \times 10^{18} \mathrm{~cm}^{-3}$ for $\mathrm{T}_{\mathrm{CH}}=15 \mathrm{~nm}$ (not shown).

The plots in Fig. $5 \& 6$ point out the following key insights: (i) Too low underlap doping results in ineffective suppression of lateral leakage (e.g. see the case with $\mathrm{N}_{\mathrm{UL}}=1 \times 10^{19} \mathrm{~cm}^{-3}$ ) (ii) Too high underlap doping, in turn, results in the source and drain regions being 'cut off' from the channel due to increased barrier height in the underlap region. This increase causes certain states in the overlap region to become quasi-bound so that they do not carry current. This results in lower ON current. Also a peculiar increase in OFF current is observed for $\mathrm{N}_{\mathrm{UL}}=2 \times 10^{19} \mathrm{~cm}^{-3}$ and $\mathrm{N}_{\mathrm{UL}}=4.5 \times 10^{19} \mathrm{~cm}^{-3}$ cases of $10 \mathrm{~nm}$ and $7.5 \mathrm{~nm}$ devices, respectively. This is caused by the induced ambipolar conduction that occurs due to WF penetration from the overlap region to the underlap region, in contrast to penetration from the drain for the no counterdoping case [6]. As seen in Fig. 7, using a too high doping could lower the barrier for hole states in the overlap regions and help them penetrate into the underlap regions controlled by the n-gate only. Moreover, highly doped regions become less sensitive to the gate control. These findings indicate that the control of the doping level is critical for the effectiveness of the counterdoping.

We also study the parameter sensitivity of the counterdoping approach by simulating cases where the border of the counterdoped region is not aligned with the actual border between the underlap and overlap regions. We denote the cases where the counterdoping extends into the overlap region with a positive value of misalignment, whereas a negative value is used for the opposite case. The results given in Fig. 8 indicate that the counterdoping method retains its effectiveness over a range of 

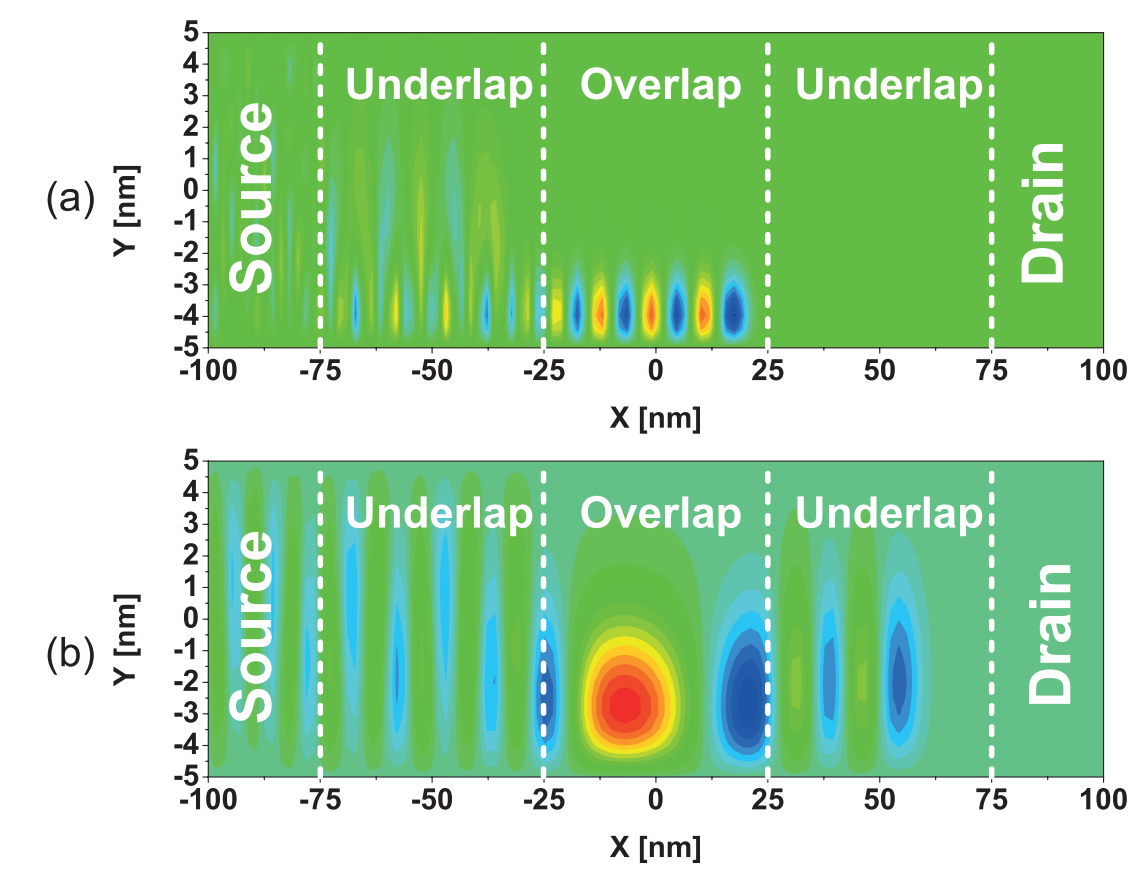

Figure 7. Heavy Hole wavefunction of the most contributing subband in the OFF state $\left(\mathrm{V}_{\mathrm{n}-\text { gate }}=-\mathrm{V}_{\mathrm{p}-\text { gate }}=1.4 \mathrm{~V}, \mathrm{~V}_{\mathrm{DS}}=0.25 \mathrm{~V}\right)$ for the counterdoping levels of (a) $\mathrm{N}_{\mathrm{UL}}=1 \times 10^{19} \mathrm{~cm}^{-3}$ (b) $\mathrm{N}_{\mathrm{UL}}=2 \times 10^{19} \mathrm{~cm}^{-3}$.

misalignments up to $-3 \mathrm{~nm}$ to $+3 \mathrm{~nm}$. We observe two different detrimental impacts on the device characteristics for negative and positive misalignment cases. For negative misalignments, the lateral leakage is not suppressed as effectively as in the perfectly aligned case; hence resulting in increased OFF current and poorer SS values. For positive misalignments, on the other hand, we observe that the ON current decreases and the SS get deteriorated due to increased barrier height in the counterdoped region.

A similar analysis has been performed varying the abruptness of the doping profiles by simulating a non-abrupt junction between the counterdoped underlap and the overlap region with various doping decay rates. As can be seen in Fig. 9, for large decay rates (>5nm/dec), an effect similar to the positive misalignment cases (i.e. as in Fig. 8) is present. Such non-abrupt doping profiles increase the potential barrier height and therefore reduce the ON current. These findings highlight the need for a very good process control in the alignment and anneal steps to ensure sufficiently abrupt junctions.

These critical aspects of the device processing can be addressed to some extent by the template-assisted selective epitaxy (TASE) growth technique recently developed [8]. This method allows horizontal epitaxial growth of fin structures by defining an oxide template. Such a method can be used to obtain the regions with alternating doping profiles with abrupt junctions that is required by the counterdoped EHBTFET without any implantation steps, which could be used for implementing the counterdoped EHBTFET on a fin. 


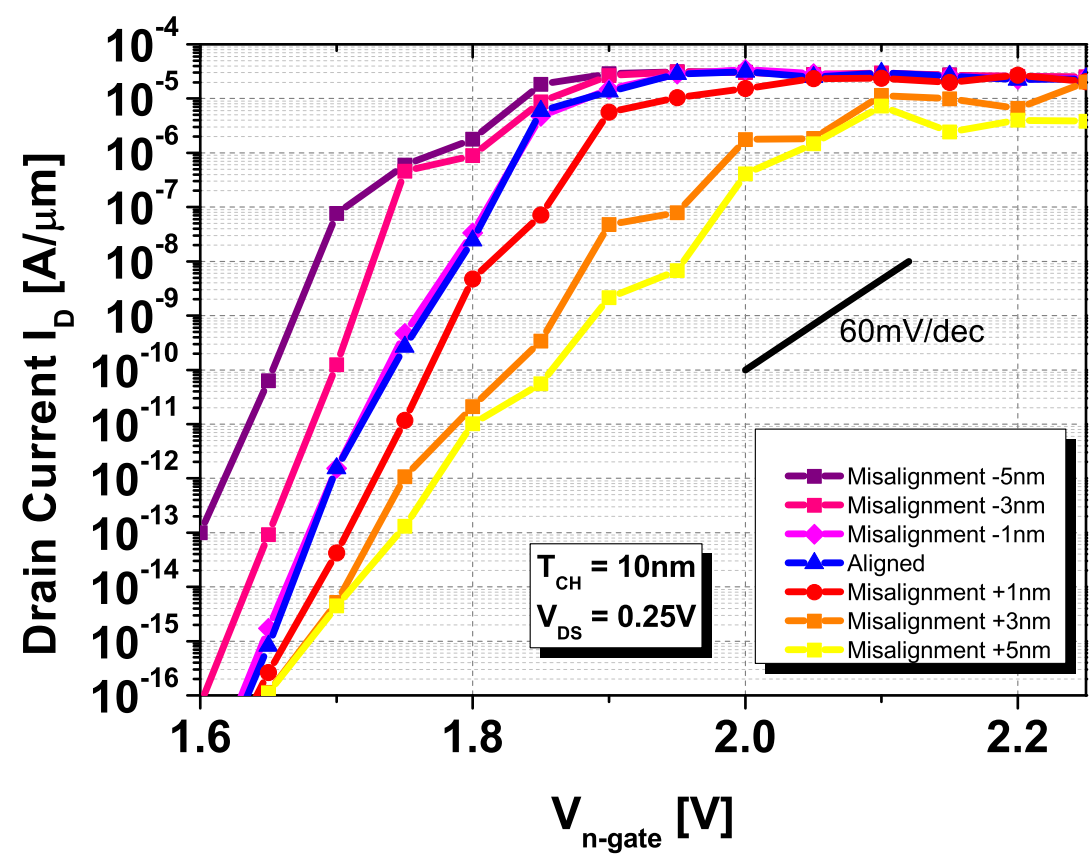

Figure 8. Transfer characteristics for the counterdoped EHBTFET $\left(\mathrm{N}_{\mathrm{UL}}=1.5 \times 10^{19} \mathrm{~cm}^{-3}\right)$ with misaligned junctions. $\left(\mathrm{V}_{\mathrm{p}-\text { gate }}=-1.4 \mathrm{~V}, \mathrm{~V}_{\mathrm{DS}}=0.25 \mathrm{~V}\right)$. Positive values of misalignment indicate the counterdoped region extends into the overlap region, whereas the negative values indicate the opposite case.

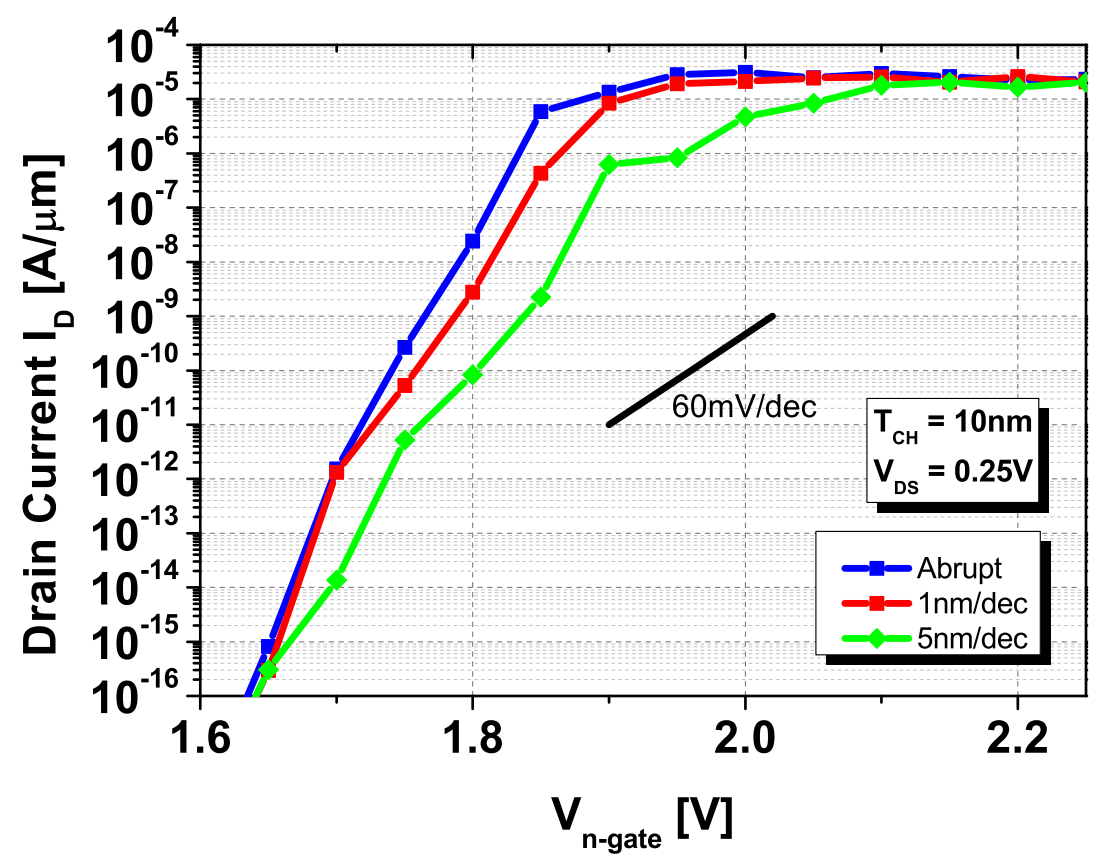

Figure 9. Transfer characteristics for the counterdoped EHBTFET $\left(\mathrm{N}_{\mathrm{UL}}=1.5 \times 10^{19} \mathrm{~cm}^{-3}\right)$ with nonabrupt junctions. $\left(\mathrm{V}_{\mathrm{p}-\text { gate }}=-1.4 \mathrm{~V}, \mathrm{~V}_{\mathrm{DS}}=0.25 \mathrm{~V}\right)$. 


\section{Conclusion}

In this work, we demonstrate the feasibility of utilizing a counterdoped structure in order to suppress the lateral leakage current using a quantum mechanical simulation method. It is shown that, depending on the body thickness and the considered biasing, there exists an optimum underlap doping value which preserves the ON current and a steep slope. It is seen that a good control of the doping profile in terms of the doping level, alignment and junction abruptness is critical for optimum performance.

\section{Acknowledgments}

The research leading to these results has received funding from the European Community's Seventh Framework Programme under grant agreement No. 619509 (Project E2-Switch). J. L. Padilla also acknowledges funding from Marie Curie Action under grant agreement No. 291780 (Andalucia Talent Hub).

\section{References}

[1] Appenzeller J, Radosavljević M, Knoch J and Avouris P 2004 Physical Review Letters 92 2-5 ISSN 0031-9007

[2] Avci U E, Morris D H and Young I A 2015 IEEE Journal of the Electron Devices Society 3 88-95 ISSN 2168-6734

[3] Krishnamohan T, Kim D, Raghunathan S and Saraswat K 2008 Double-Gate Strained-Ge Heterostructure Tunneling FET (TFET) With record high drive currents and $<60 \mathrm{mV} / \mathrm{dec}$ subthreshold slope 2008 IEEE International Electron Devices Meeting vol 67 (IEEE) pp 1-3 ISBN 978-1-4244-2377-4 ISSN 01631918

[4] Lattanzio L, De Michielis L and Ionescu A M 2011 Electron-hole bilayer tunnel FET for steep subthreshold swing and improved ON current 2011 Proceedings of the European Solid-State Device Research Conference (ESSDERC) (IEEE) pp 259-262 ISBN 978-1-4577-0707-0

[5] Alper C, Lattanzio L, De Michielis L, Palestri P, Selmi L and Ionescu A M 2013 IEEE Transactions on Electron Devices 60 2754-2760 ISSN 0018-9383

[6] Alper C, Palestri P, Lattanzio L, Padilla J L and Ionescu A M 2014 Two dimensional quantum mechanical simulation of low dimensional tunneling devices 2014 44th European Solid State Device Research Conference (ESSDERC) 1 (IEEE) pp 186-189 ISBN 978-1-4799-4376-0

[7] Padilla J L, Alper C, Gámiz F and Ionescu A M 2014 Applied Physics Letters 105082108 ISSN 0003-6951

[8] Schmid H, Borg M, Moselund K, Gignac L, Breslin C M, Bruley J, Cutaia D and Riel H 2015 Applied Physics Letters 106233101 ISSN 0003-6951

[9] Trellakis A, Galick A T, Pacelli A and Ravaioli U 1997 Journal of Applied Physics 817880 ISSN 00218979

[10] Schenk A, Stahl M and Wünsche H J 1989 physica status solidi (b) 154 815-826 ISSN 03701972

[11] Alper C, Palestri P, Padilla J L, Gnudi A, Grassi R, Gnani E, Luisier M and Ionescu A M 2015 Efficient quantum mechanical simulation of band-to-band tunneling EUROSOI-ULIS 2015: 2015 Joint International EUROSOI Workshop and International Conference on Ultimate Integration on Silicon pp 141-144 ISBN 978-1-4799-6911-1

[12] Alper C, Palestri P, Lattanzio L, Padilla J and Ionescu A 2015 Solid-State Electronics 113 167-172 ISSN 00381101

[13] Yamanishi M and Suemune I 1984 Jpn. J. Appl. Phys. 23 L35-L36 ISSN 0021-4922

Published in Semic. Sci tech, v.31, n.4, p.045001 
[14] Alper C, Visciarelli M, Palestri P, Padilla J L, Gnudi A, Gnani E and Ionescu A M 2015 Modeling the imaginary branch in III-V tunneling devices: Effective mass vs $k \cdot p 2015$ International Conference on Simulation of Semiconductor Processes and Devices (SISPAD) (IEEE) pp 273-276 ISBN 978-1-4673-7858-1 URL http://ieeexplore. ieee.org/lpdocs/epic03/wrapper.htm?arnumber $=7292312$

[15] Sabathil M 2004 Opto-electronic and quantum transport properties of semiconductor nanostructures Ph.D. thesis

[16] Goetz K H, Bimberg D, Jurgensen H, Selders J, Solomonov a V, Glinskii G F and Razeghi M 1983 Journal of Applied Physics 544543 ISSN 00218979

[17] Goldberg Y A and NM S 1999 Handbook Series on Semiconductor Parameters (World Scientific Publishing Co. Pte. Ltd.) ISBN 9789812832078

[18] Sire C, Blonkowski S, Gordon M J and Baron T 2007

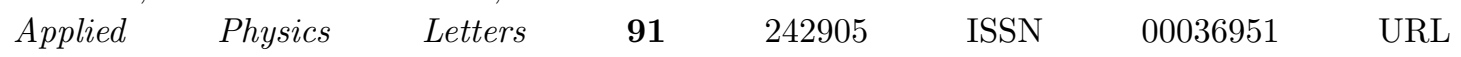
http://scitation.aip.org/content/aip/journal/apl/91/24/10.1063/1.2822420 